\title{
Signifikanter Vorteil der Erhaltungstherapie
}

Moderne Therapien haben das Überleben von Patienten mit Multiplem Myelom in den vergangenen Jahren deutlich verlängert. Für neu diagnostizierte Patienten, die für eine Autologe Stammzelltransplantation (ASCT) geeignet sind, zeigt eine aktuelle Metaanalyse nun auch einen signifikanten Vorteil des Immunmodulators Lenalidomid in der Erhaltungstherapie für das Gesamtüberleben und das progressionsfreue Überleben. Die Bedeutung der Ergebnisse spiegelt sich auch in der bevorzugten Publikation als „Rapid Communication" im Journal of Clinical Oncology wider [1].

Lenalidomid moduliert die Immunantwort, hemmt das Wachstum des Multiplen Myeloms und eignet sich für die Erhaltungstherapie nach ASCT, stellen die Autoren fest. Im Februar diesen Jahres wurde Lenalidomid (Revlimid $^{\oplus}$ ) in Europa für die Erhaltungstherapie bei neu diagnostizierten Patienten mit Multiplem Myelom nach einer autologen Stammzelltransplantation zugelassen. Die Zulassung basierte auf den Daten der multizentrischen, randomisierten, kontrollierten, doppelblinden Phase III-Studien CALGB und IFM. Die nun veröffentlichte Metaanalyse erfasste auch die Daten der offenen, multizentrischen Phase II-Studie GINMEA RVMM-PI-209 mit insgesamt 1208 Patienten mit neu diagnostiziertem Multiplem Myelom (NDMM) und zeigte die Überlegenheit von Lenalidomid in der Erhaltungstherapie gegenüber Placebo oder keiner Erhaltungstherapie.

\section{Prozent niedrigeres Mortalitätsrisiko}

Nach einer medianen Nachbeobachtungszeit aller überlebenden $\mathrm{Pa}$ tienten, wurde das mediane OS für die Gruppe mit Lenalidomid Erhaltungstherapie noch nicht erreicht, verglichen mit 86,0 Monaten in der Kontrollgruppe. Dies bedeutet eine signifikante Reduktion des Mortalitätsrisikos um 25 Prozent für die Lenalidomid Erhaltungstherapie verglichen mit der Kontrollgruppe. Die 7-Jahres Überlebensrate betrug mit Lenalidomid Erhaltungstherapie 62 Prozent verglichen mit 50 Prozent in der Kontrollgruppe zum Stichtag 1. März 2015. Analysen zu einem späteren Stichtag
2016 zeigten ein medianes Überleben von 111 Monaten (9,25 Jahren) mit Lenalidomid Erhaltungstherapie versus 86,9 Monaten (7,2 Jahren) in der Kontrollgruppe.

Der zuvor bereits in Einzelstudien beobachtete Vorteil im progressionsfreien Überleben (PFS) mit Lenalidomid wurde in der Metaanalyse in jeder einzelnen der drei Studien und in allen Subgruppen bestätigt. Die Gesamtanalyse der Daten ergab ein PFS in der Lenalidomid Gruppe von 52,8 Monaten gegenüber 23,5 Monaten in der Kontrollgruppe. Das Risiko für eine Krankheitsprogression oder Tod wurde mit der Lenalidomid Erhaltungstherapie gegenüber der Kontrollgruppe um 52 Prozent reduziert. Ebenso zeigte sich beim explorativen Endpunkt PFS2, der als Zeitspanne von der Randomisierung bis zu einem sekundären Progressionsereignis oder dem Tod definiert war, ein Vorteil für die Lenalidomid Erhaltungstherapie mit einem medianen PFS2 von 73,3 Monaten (6,1 Jahren) verglichen mit 56,7 Monaten (4,7 Jahren) in der Kontrollgruppe.

Keine der Studien untersuchte eine vorbestimmte Dauer der Erhaltungstherapie stellen die Autoren der Metaanalyse fest. Daher wäre in künftigen Studien interessant, unterschiedliche Zeitspannen für die Erhaltungstherapie zu evaluieren, speziell bei Patienten, die einen negativen Status einer Minimalen Residualerkrankung aufweisen.

\section{Neue Standardtherapie für Patienten nach ASCT}

Die Europäische Gesellschaft für Klinische Onkologie (ESMO) zitiert die Da- ten der Metanalyse in den aktuellen klinischen Praxisrichtlinien für das Multiple Myelom und hebt dabei den Vorteil einer Lenalidomid Erhaltungstherapie für das Gesamtüberleben hervor. Lenalidomid ist nun die einzige zugelassene Therapie für die Erhaltungstherapie von NDMM-Patienten nach ASCT sowohl in Europa als auch in den USA. Ein wichtiges Therapieziel für Patienten mit NDMM ist die Erreichung oder der Erhalt einer Langzeitkrankheitskontrolle. Die Erhaltungstherapie nach ASCT kann als wirksamer Ansatz zur Erreichung einer solchen Langzeitkontrolle der Erkrankung betrachtet werden, wodurch die Zeit für einen Rückfall und eine Zweitlinienbehandlung hinausgezögert und das Überleben verlängert werden kann. Im Lichte neuer, hochaktiver Dreifach-Induktionstherapie-Regime, die die Tiefe und Dauer des Ansprechens verbessern, ebenso wie derzeit laufende Studien, die den optimalen Zeitpunkt für ASCT untersuchen, könne die Erhaltungstherapie mit Lenalidomid für Patienten, die sich für eine ASCT eignen, als Standardbehandlung betrachtet werden.

\section{Literatur \\ 1. McCarthy PL et al (2017) Lenalidomide \\ Maintenance After Autologous Stem-Cell \\ Transplantation in Newly Diagnosed Multip- \\ le Myeloma: A Meta-Analysis. J Clin Oncol 35}

Wien klin Mag 2017 · 20:145

DOI 10.1007/s00740-017-0188-8

C Springer-Verlag GmbH Austria 2017
Quelle: Presseaussendung Celgene $\mathrm{GmbH}$ 